\title{
StudentKost: a cross-sectional study assessing college students' diets: reason for concern?
}

\author{
Erlend L. Valen* (1), Dagrun Engeset, Nina C. Øverby and Elisabet R. Hillesund \\ Department of Nutrition and Public Health, Faculty of Health and Sport Sciences, University of Agder, PO Box 422 , 4604 Kristiansand, Norway
}

(Received 17 July 2020 - Accepted 24 July 2020)

Journal of Nutritional Science (2020), vol. 9, e39, page 1 of 9

doi:10.1017/jns.2020.33

Abstract

College students constitute a significant proportion of the young adult population in Norway. They are in their reproductive years, which is of interest regarding diet and preconception health. Our objective was to assess young college students' diet and nutrient intake in relation to national dietary recommendations and assess the probability of inadequate micronutrient intake for both genders using the Nordic Nutrition Recommendations, and also to evaluate its consequences on preconception health and create a groundwork for future interventions on this group. At the University of Agder (UiA), we enrolled 622 students aged 18-40 years for a cross-sectional study of student's diet, StudentKost. The students completed a food frequency questionnaire, including questions of supplement use, over the past 4 weeks. Intake of fruits, vegetables, oily fish, and whole grain was lower than recommended, as were mean intake of folate, iron, and iodine. Our main findings are that students have a somewhat suboptimal diet compared to the Norwegian dietary guidelines. Male students had generally lower diet quality than females. Compared to the Nordic Nutrition Recommendations (NNR), we also saw a relatively high probability of inadequate intake of several micronutrients and a very high probability for some micronutrients in a significant portion of the sample. Public health effort should be directed towards improving students and young adults' diet in general, and interventions towards improving preconception health should be explored. The low participation rate limits the generalizability of our findings. Our findings encourage further investigation into young adults' diet.

Key words: Norway: Food consumption survey: Dietary intake: Young adults: Preconception health

\section{Introduction}

Diet strongly influences human health. Over the years, there has been a shift from exploring deficient intake of single nutrients to explore the importance of overall diet for human development and health ${ }^{(1)}$. Based on this research, evidence-based national food-based dietary guidelines have been developed, as well as age- and gender-specific recommendations for nutrient intake. The Norwegian health authorities survey population diet at regular intervals. Norkost 3, a nation-wide dietary survey carried out in 2011, indicated that the adult Norwegian population mainly had nutrient intakes within recommendations, but that a relatively large proportion did not comply with the dietary guidelines for many of the core food groups ${ }^{(2)}$. In general, there has been limited research on young adults' diet in Norway, and Norkost 3 from 2011 is the only nation-wide food consumption survey that has been completed on adults since the Norkost 2 survey in $1997^{(3)}$

More knowledge regarding young adults' diet and dietary behaviour is warranted because new research has demonstrated associations between lifestyle and diet before conception and subsequent children's development and health ${ }^{(4)}$. The focus has mainly been on the mothers' diet during pregnancy as the first step in affecting child health. Recently, the focus is expanded to include adolescents and young adults' diet in the years and months before parenthood ${ }^{(4-6)}$. Which again is leading to increased awareness around a topic that has barely been addressed, i.e. how fathers' diet and behaviour before conception can affect their children's health. Paternal

*Corresponding author: Erlend L. Valen, email erlend.l.valen@uia.no

(C) The Author(s), 2020. Published by Cambridge University Press on behalf of The Nutrition Society. This is an Open Access article, distributed under the terms of the Creative Commons Attribution licence (http://creativecommons.org/licenses/by/4.0/), which permits unrestricted re-use, distribution, and reproduction in any medium, provided the original work is properly cited. 
obesity and undernutrition are both associated with increased DNA damage and endocrine misregulations ${ }^{(5)}$, while sperm motility could be positively affected by a healthy $\operatorname{diet}^{(7)}$. According to Frey et al., improving men's preconception health is important in conjunction with a healthy pregnancy, both directly and indirectly, including improving pregnancy outcomes and health practices as well as improving capacity for parenthood $^{(8)}$.

Although a healthy diet is especially important during pregnancy to ensure a sufficient intake of vital micronutrients ${ }^{(9)}$, an optimal micronutrient status should ideally be acquired in advance $^{(4)}$. Stephenson et al. are concerned that many women in low-, medium- and high-income countries will not be nutritionally prepared for pregnancy ${ }^{(4)}$. Public health interventions targeting dietary behaviour ahead of pregnancy may be a path to proceed. Barker et al. argue that although it is too early to draw definite conclusions about the long-term effect of pre-pregnancy diet interventions, the potential health benefits will be so high, and the costs so low, that research on measures on how to promote this should be explored ${ }^{(6)}$.

College students constitute a significant proportion of the young adult population, making up one-third of people aged 19-24 in Norway ${ }^{(10)}$. Many students are in a dietary transition phase moving away from home and establishing their own family. There is limited knowledge about college students' diet and dietary behaviours in Norway. This knowledge is needed to substantiate the potential need for interventions targeting diet and lifestyle in preconception years.

The objective of this study was to assess students' diet in relation to official dietary guidelines provided by the Norwegian Nutrition Council ${ }^{(1)}$ and the Nordic Nutrition Recommendations ${ }^{(11)}$. Further, we aimed to provide assessments of the probability of inadequate micronutrient intake among men and women according to recommended intake (RI), AR and LI, hopefully, helping to identify a potential need and creating a foundation, for interventions to improve diet in this population group during the preconception period.

\section{Study design}

This cross-sectional study, called StudentKost, involved a sample of students from the University of Agder, Norway, aged between 18 and 40 years. The average age for men at first birth was 31.8 years in Norway in 2018 and has been above 30 since 1999, so to take this into account we increased the inclusion criteria to 40 years ${ }^{(12)}$. Our recruitment period lasted from the end of October to the beginning of December in 2018. We aimed to recruit 1000 students. In order to aid recruitment, we produced and presented a short promotional video at the university canteens, as well as distributing it in relevant social media. We also distributed flyers in university classrooms and group study rooms. Due to the limited response rate from these recruitment methods, we obtained permission from the university leadership to send out invitations to the university students' institutional email. The emailed invitation described the study and included a link to the study's web-page, where potential participants could agree to participate and complete the web-based survey. The survey included a validated food frequency questionnaire ${ }^{(13)}$, which was used to assess habitual dietary intake. SurveyXact, a web-based tool for conducting questionnaire-based programs, was used to conduct the survey ${ }^{(14)}$.

\section{Method}

The questionnaire consisted of 209 questions divided into five categories: general information about the participant (5 items), food frequency questions (152 items), eating habits (12 items), motivation and barriers (26 items), physical activity and sedentary behaviour ( 8 items), and tobacco use (6 items). The general information comprised questions about gender, age, height, weight and education. The food frequency part of the questionnaire divided, e.g. beverages, bread, main courses, fruit and vegetables, into different categories. The response alternatives given in the survey ranged from never eating the specific food item, to eating it every day. There were in total five to seven different frequency categories depending on the nature of the question. The participants had to answer every question in the questionnaire to continue, except questions on height, weight and their email address. The test-retest reproducibility and validity of the Food frequency questionnaire (FFQ) have been assessed and found to have a fair relative validity ${ }^{(13)}$.

Two different programs were used to calculate nutrient intake from the questionnaire, i.e. Python ${ }^{(15)}$ and FoodCalc ${ }^{(16)}$. Python calculates the quantity of each registered food item for every participant in the questionnaire. FoodCalc estimates the nutrient intake by pairing the registered quantity of each food item with its nutrient content from the relevant food item code given in the Norwegian Food Composition Table. This Table provides information concerning the content of nutrients and energy in the most commonly consumed foods in Norway ${ }^{(17)}$. Food intakes are given in grams per day while results for nutrient intakes are presented in grams, milligrams, micrograms or as a percentage of total energy intake depending on the variable of interest. The nutritional calculation does not include supplement use.

Absolute food intakes were compared to official dietary guidelines and the Nordic Nutrition Recommendation (NNR) for the adequacy of nutrient intakes ${ }^{(18)}$. The NNR is based on a comprehensive collaboration of more than 100 experts who reviewed countless research findings in order to determine specific health outcomes of different foods and dietary patterns ${ }^{(11)}$. These assessments have underpinned the national dietary advice, which is used as a reference point in this survey.

\section{Adequacy of micronutrient intake}

Adequacy of micronutrient intake was assessed according to three measures provided by the NNR: the proportion of participants exceeding RI, the proportion of participants with intake below the average requirement (AR) and the proportion of participants with calculated intake below lower intake (LI). $\mathrm{RI}$ is estimated to cover individual requirements for $97-98 \%$ of the age- and gender-specific population; hence, intakes 
above this value imply a low probability of inadequate intake. $\mathrm{AR}$ is estimated to cover the requirement for half of the ageand gender-specific population. Intake below this value for a specific micronutrient implies a relatively high probability of inadequate intake ${ }^{(11)}$. LI represents a lower limit of micronutrient intake below which the probability of inadequate intake is very high. Of note, the intake of a specific micronutrient between AR and RI does not guarantee meeting individual needs.

\section{Statistical analysis}

We used IBM SPSS 24.0 to analyse all the data ${ }^{(19)}$. All analyses were carried out stratified by gender, inspecting each variable for deviance from a normal distribution. For comparisons of diet across gender, we used independent sample T-tests, or Mann-Whitney $U$ tests depending on the distribution. The significance level was set to $P<0 \cdot 05$.

\section{Results}

Whereas the invitation was sent to all university students (approximately 13 000), our goal was 1000 participants. A total of 743 participants responded and answered at least one or more questions, equalling to $74 \%$ of our initial goal. Out of these, 617 completed the entire survey. Upon a closer examination of the response patterns among non-completers, we included five who answered each question in the food frequency section of the questionnaire so that their data could be calculated for food and nutrient intake. The final sample included in our analysis was 622. No participants were excluded from the analysis, as no one had an implausible energy intake.

The proportions between women and men in the survey were 71 and $29 \%$, respectively, with a mean age of 23.4 years for women, and 23.6 years for men. Table 1 shows height, weight, body mass index (BMI) and educational attainment for the participants. The average BMI was $23 \cdot 8 \mathrm{~kg} / \mathrm{m}^{2}$ for women and $24.4 \mathrm{~kg} / \mathrm{m}^{2}$ for men. A total of $29 \%$ female and $33 \%$ male respondents were classified with overweight or obesity. In total, $51 \%$ women and $52 \%$ men in this survey had studied for at least 1 year at a university, meaning that a large proportion of the sample were first-time students.

\section{Food consumption}

The questionnaire covered a substantial number of food items that were grouped into broader categories for analysis. Table 2 presents the food categories, including fruit, vegetables, fish, whole grain, meat and dairy products. Median reported intake of fruit and vegetables was $209 \mathrm{~g} / \mathrm{d}$ for women and $148 \mathrm{~g} / \mathrm{d}$ for men, which is less than half of the recommended $500 \mathrm{~g} / \mathrm{d}$. With juice included, the numbers improved to $244 \mathrm{~g} / \mathrm{d}$ for women and $186 \mathrm{~g} / \mathrm{d}$ for men. Women had significantly higher fruit and vegetable intake than men in the both cases. For vegetables, $8 \%$ of women met the recommended intake and $5 \%$ of men, while $16 \%$ of women and $8 \%$ of men met the recommendations for fruit intake. For fruits and vegetables combined, a total of $5 \%$ women and $2 \%$ of men met both recommendations; by including juice, this increased to 14 and $9 \%$ for women and men, respectively.

Mean fish intake was $225 \mathrm{~g} /$ week for women and $238 \mathrm{~g} /$ week for men, with oily fish contributing $115 \mathrm{~g} /$ week for both genders. This is substantially lower than the recommended 300-450 g/week of fish in general, and $200 \mathrm{~g} /$ week of oily fish. Approximately $17 \%$ did not report eating any fish. Additionally, both genders had a lower-thanrecommended intake of $70-90 \mathrm{~g} / \mathrm{d}$ of whole-grain products; women consumed $67 \mathrm{~g} / \mathrm{d}$, while men consumed $59 \mathrm{~g} / \mathrm{d}$. The consumption of red meat added up to $53 \mathrm{~g} / \mathrm{d}$ for women and $78 \mathrm{~g} / \mathrm{d}$ for men. Men ate significantly more red meat than women. It is recommended to limit the consumption of red and processed meat to less than $500 \mathrm{~g} /$ week or approximately $70 \mathrm{~g} / \mathrm{d}$. Around $17 \%$ of women in this sample did not consume red meat, while less than $2 \%$ of men did the same. A total of $2.3 \%$ women and $1.7 \%$ of men did not eat any kind of meat. Total milk intake added up to $71 \mathrm{ml} / \mathrm{d}$ for women and $78 \mathrm{ml} / \mathrm{d}$ for men.

\section{Energy intake}

Table 3 presents energy intake and macronutrient contribution. Mean energy intake was $8.6 \mathrm{MJ} / \mathrm{d}$ for women and 9.5 $\mathrm{MJ} / \mathrm{d}$ for men. On average, carbohydrates contributed to $44.5 \mathrm{E} \%$ for women, of which $5.8 \mathrm{E} \%$ came from added sugar. For men, energy from carbohydrates amounted to $42.1 \mathrm{E} \%$, of which $5.9 \mathrm{E} \%$ came from added sugar. Thus, both genders had sugar intake well within the recommended limit of $10 \mathrm{E} \%$. The main contributor to sugar intake was soda, with a median intake of $35.7 \mathrm{ml} / \mathrm{d}$ for both genders. Dietary fibre intake was lower than the recommended 25 $35 \mathrm{~g} / \mathrm{d}$ for both genders but significantly higher for women than for men $(22 \cdot 1$ v. $20 \cdot 3 \mathrm{~g} / \mathrm{d})$.

Fat contributed on average $33.4 \mathrm{E} \%$ for women and $34.3 \mathrm{E}$ $\%$ for men. Both genders met the recommended intake of omega 3 and 6 of at least $3 \%$ of the total energy intake combined. Saturated fatty acids contributed to $12 \cdot 1 \mathrm{E} \%$ for women and $12.7 \mathrm{E} \%$ for men which is higher than the recommended upper limit of $10 \mathrm{E} \%$ for both genders. Monounsaturated fats contributed to $12.9 \mathrm{E} \%$ for women and $13.3 \mathrm{E} \%$ for men. Polyunsaturated fats made up $5.0 \mathrm{E}$ $\%$ for both genders, which is at the lower end of the recommended 5-10 E\%. Protein contributed to a total of 17.4 E $\%$ for women and $17.9 \mathrm{E} \%$ for men, which is within the recommended range of 10-20 E\%. Finally, alcohol amounted to an average of $1.5 \mathrm{E} \%$ for women and $3.4 \mathrm{E} \%$ for men. In this sample, $16.7 \%$ reported not drinking any alcohol.

\section{Micronutrients}

Tables 4 and 5 present mean and median micronutrient intake compared to the recommended intake (RI), the average requirement (AR) and lower intake level (LI) for both genders, respectively. On average, men had a significantly higher intake of most micronutrients compared to that of women. However, as the recommended intake is higher for men regarding most micronutrients, intake was still suboptimal for some nutrients. 
Table 1. Age, height, weight, BMI and educational attainment among participating women and men in the StudentKost study

\begin{tabular}{|c|c|c|c|c|c|c|c|c|}
\hline & \multicolumn{4}{|c|}{ Women $\left(n 444^{\mathrm{a}}\right)$} & \multicolumn{4}{|c|}{ Men ( $n$ 178) } \\
\hline & Mean & SD & Median & 25,75 & Mean & SD & Median & 25,75 \\
\hline Age, years & 23.4 & 4.5 & $22 \cdot 0$ & $20 \cdot 0,24 \cdot 0$ & 23.6 & 4.0 & 23.0 & $21 \cdot 0,25 \cdot 0$ \\
\hline Height, cm & $168 \cdot 3$ & $6 \cdot 4$ & 168.0 & $164.0,173.0$ & 182.4 & 7.4 & 183.0 & $178.0,187.0$ \\
\hline Weight, kg & $67 \cdot 4$ & $12 \cdot 7$ & 65.0 & $59 \cdot 0,74.0$ & $81 \cdot 2$ & $14 \cdot 1$ & 80.0 & $72 \cdot 0,89 \cdot 0$ \\
\hline \multirow[t]{4}{*}{ BMI, $\mathrm{kg} / \mathrm{m}^{2}$} & 23.8 & $4 \cdot 2$ & $23 \cdot 3$ & $21 \cdot 2,25 \cdot 6$ & $24 \cdot 4$ & 3.6 & 23.9 & $22 \cdot 2,25 \cdot 9$ \\
\hline & & & & Educatio & ttainme & & & \\
\hline & Women & & & & Men & & & \\
\hline & $n$ & & $\%$ & & $n$ & & $\%$ & \\
\hline Primary school & 3 & & 0.7 & & 0 & & - & \\
\hline Secondary school & 197 & & 44.4 & & 70 & & 39.3 & \\
\hline Vocational secondary school & 15 & & 3.4 & & 14 & & 7.9 & \\
\hline University, less than 4 years & 154 & & 34.7 & & 74 & & 41.6 & \\
\hline University, more than 4 years & 71 & & $16 \cdot 0$ & & 19 & & $10 \cdot 7$ & \\
\hline Other education & 4 & & 0.9 & & 1 & & 0.6 & \\
\hline
\end{tabular}

${ }^{a}$ Weight is missing 9 ( $n$ 435) values, and height is missing 2 ( $n$ 442) values.

Table 2. Intake of different foods and their respective recommendations, in median $\mathrm{g} / \mathrm{d}$, if not otherwise stated

Foods, $g / d$

\begin{tabular}{|c|c|c|c|c|c|}
\hline & \multicolumn{2}{|c|}{ Women } & \multicolumn{2}{|c|}{ Men } & \multirow[b]{2}{*}{ Recommendation $^{a}$} \\
\hline & Median & 25,75 & Median & 25,75 & \\
\hline Fish, g/week & $225 \cdot 0$ & $75 \cdot 0,397.5$ & 237.5 & $75.0,375.0$ & $300-450 \mathrm{~g} /$ week \\
\hline Oily fish, g/week & $115 \cdot 0$ & $40 \cdot 0,190 \cdot 0$ & $115 \cdot 0$ & $40 \cdot 0,190 \cdot 0$ & $200 \mathrm{~g} /$ week \\
\hline White fish, g/week & $100 \cdot 0$ & $0.0,175 \cdot 0$ & 87.5 & $0.0,175 \cdot 0$ & - \\
\hline Whole grain ${ }^{\mathrm{b}}$ & 66.6 & $38.6,110 \cdot 1$ & $59 \cdot 1$ & $37 \cdot 8,96 \cdot 6$ & $70-90 \mathrm{~g} / \mathrm{d}$ \\
\hline Red meat $^{\mathrm{c}}$ & $52 \cdot 7$ & $27 \cdot 0,80 \cdot 6$ & $78 \cdot 4^{\star \star \star}$ & $51 \cdot 1,115 \cdot 4$ & Limit consumption to $<500 \mathrm{~g} /$ week (approx. $70 \mathrm{~g} / \mathrm{d}$ ) \\
\hline Fruit & $99 \cdot 1^{\star \star \star}$ & $50 \cdot 8,174.9$ & 59.9 & $28 \cdot 9,126 \cdot 2$ & $250 \mathrm{~g} / \mathrm{d}$ \\
\hline Vegetables & $104 \cdot 9^{\star \star *}$ & $59 \cdot 5,162 \cdot 3$ & $74 \cdot 3$ & $35 \cdot 9,116 \cdot 8$ & $250 \mathrm{~g} / \mathrm{d}$ \\
\hline Fruit and vegetables & $208 \cdot 9^{\star \star \star}$ & $133 \cdot 5,331.5$ & $148 \cdot 0$ & $82 \cdot 5,233 \cdot 3$ & $500 \mathrm{~g} / \mathrm{d}$ \\
\hline Juice, orange- or apple-, ml & $14 \cdot 3$ & $0 \cdot 0,57 \cdot 1$ & $14 \cdot 3$ & $0 \cdot 0,57 \cdot 1$ & $200 \mathrm{ml}$ \\
\hline Fruit and vegetables, including juice & $243 \cdot 9^{\star \star *}$ & $160 \cdot 9,387 \cdot 7$ & 185.7 & $107 \cdot 0,288 \cdot 8$ & $500 \mathrm{~g} / \mathrm{d}$ \\
\hline Total milk, ml & 71.4 & $26 \cdot 8,199 \cdot 6$ & $77 \cdot 7^{\star}$ & $28 \cdot 6,227 \cdot 2$ & - \\
\hline Energy-dense dairy products & $18 \cdot 8$ & $9 \cdot 3,33 \cdot 3$ & $22 \cdot 8^{*}$ & $9 \cdot 1,50 \cdot 0$ & Reduce intake \\
\hline Egg & $7 \cdot 0$ & $0.0,17 \cdot 6$ & $17 \cdot 6$ & $0 \cdot 0,35 \cdot 2$ & - \\
\hline
\end{tabular}

The StudentKost study.

${ }^{a}$ Dietary recommendations from the Norwegian Nutrition Council ${ }^{(1)}$.

${ }^{b}$ Calculated using data from the Norwegian Nutrition Council ${ }^{(1)}$, product web sites ${ }^{(20)}$, and the Norwegian Food Composition Table ${ }^{(21)}$

${ }^{c}$ Calculated using information from manufacturers websites ${ }^{(22-26) .}$

Significance level for differences between genders: ${ }^{\star} P<0.05,{ }^{\star \star \star} P<0.001$

Table 3. Energy intake and macronutrient contribution to energy intake compared to the Nordic Nutrition Recommendations by gender, presented as E\% if not otherwise stated.

\begin{tabular}{|c|c|c|c|c|c|c|c|c|c|}
\hline & \multicolumn{4}{|c|}{ Women } & \multicolumn{4}{|c|}{ Men } & \multirow[b]{2}{*}{ NNR (E\%) } \\
\hline & Mean & SD & Median & 25,75 & Mean & SD & Median & 25,75 & \\
\hline Energy, kcal & 2045 & 719 & 1993 & 1535,2376 & 2274 & $739^{\star \star \star}$ & 2203 & 1736,2780 & - \\
\hline Energy, $\mathrm{MJ} / \mathrm{d}$ & 8.6 & 3.0 & $8 \cdot 3$ & $6 \cdot 4,10 \cdot 0$ & 9.5 & $3 \cdot 1^{*}$ & 9.2 & $7 \cdot 3,11 \cdot 7$ & - \\
\hline Carbohydrates & 44.5 & $6 \cdot 7^{\star \star \star *}$ & $44 \cdot 2$ & $40 \cdot 5,48 \cdot 3$ & $42 \cdot 1$ & $7 \cdot 1$ & 41.9 & $38.3,45.9$ & $45-60$ \\
\hline Added sugar & 7.1 & $5 \cdot 2$ & 5.8 & $3.9,8.4$ & $7 \cdot 0$ & 5.5 & 5.9 & $3.4,8.7$ & $<10$ \\
\hline Dietary fibre, $g / d$ & 23.7 & $10 \cdot 6$ & $22 \cdot 1$ & $15 \cdot 7,29 \cdot 2^{*}$ & 21.9 & $11 \cdot 2$ & $20 \cdot 3$ & $14 \cdot 7,26 \cdot 8$ & $25-35 \mathrm{~g} / \mathrm{d}$ \\
\hline Total fat & 33.4 & 5.6 & 33.5 & $30,36 \cdot 9$ & 34.3 & 5.8 & 34.3 & $30 \cdot 7,38 \cdot 1$ & $25-40$ \\
\hline SFA & $12 \cdot 1$ & 3.0 & $12 \cdot 1$ & $10 \cdot 3,14 \cdot 0$ & $12 \cdot 7$ & $3 \cdot 2^{*}$ & 12.5 & $10 \cdot 5,14 \cdot 2$ & $<10$ \\
\hline TFA & 0.3 & 0.1 & 0.3 & $0.2,0.4$ & 0.3 & 0.2 & 0.3 & $0.2,0.4$ & As low as possible \\
\hline MUFA' & $12 \cdot 9$ & 2.4 & $12 \cdot 7$ & $11 \cdot 3,14.4$ & $13 \cdot 3$ & 2.5 & $13 \cdot 1$ & $11 \cdot 6,15 \cdot 1$ & $10-20$ \\
\hline PUFA & 5.0 & $1 \cdot 1$ & 4.9 & $4 \cdot 3,5 \cdot 7$ & $5 \cdot 0$ & $1 \cdot 3$ & 4.8 & $4 \cdot 1,5 \cdot 7$ & $5-10$ \\
\hline Omega 3 & $1 \cdot 1$ & 0.4 & 1.0 & $0.8,1.3$ & $1 \cdot 1$ & 0.5 & 1.0 & $0.7,1.4$ & At least $3 \mathrm{E} \%$ \\
\hline Omega 6 & 3.8 & 0.8 & 3.8 & $3 \cdot 3,4.4$ & 3.8 & 0.9 & 3.7 & $3 \cdot 2,4 \cdot 4$ & \\
\hline Protein & $17 \cdot 4$ & $3 \cdot 1$ & $17 \cdot 3$ & $15 \cdot 3,19 \cdot 3$ & 17.9 & 3.9 & $17 \cdot 6$ & $15 \cdot 8,19 \cdot 6$ & $10-20$ \\
\hline Alcohol & 2.4 & 2.5 & 1.5 & $0.6,3.5$ & 3.8 & 3.8 & 3.4 & $0.7,6 \cdot 0^{\star \star \star}$ & $<5$ \\
\hline
\end{tabular}

The StudentKost study.

NNR, Nordic Nutrition Recommendations; SFA, saturated fatty acids; TFA, trans fatty acids; 'MUFA, monounsaturated fatty acids; PUFA, polyunsaturated fatty acids.

Significance level for differences between genders: ${ }^{\star} P<0.05,{ }^{\star \star \star} P<0.001$. 
Table 4. Micronutrient intake per day compared to NNR and related to recommendations in percentage.

Women

\begin{tabular}{|c|c|c|c|c|c|c|c|c|c|c|}
\hline \multirow[b]{2}{*}{ Micronutrients } & \multirow[b]{2}{*}{ Mean } & \multirow[b]{2}{*}{ SD } & \multirow[b]{2}{*}{ Median } & \multirow[b]{2}{*}{ 25th-75th percentile } & \multicolumn{3}{|c|}{ NNR2012 recommendation } & \multicolumn{3}{|c|}{ Percentage of participants ${ }^{a}$} \\
\hline & & & & & $\mathrm{RI}^{\mathrm{b}}$ & $\mathrm{AR}^{\mathrm{b}}$ & $\mathrm{LI}^{\mathrm{b}}$ & $\geq \mathrm{RI}$ & $<\mathrm{AR}$ & $<\mathrm{LI}$ \\
\hline Vitamin A, $\mu \mathrm{g}$ & 930 & 763 & 715 & 463,1089 & $700 \mu \mathrm{g}$ & $500 \mu \mathrm{g}$ & $400 \mu \mathrm{g}$ & $52 \%$ & $29.5 \%$ & $16 \%$ \\
\hline Vitamin $D, \mu \mathrm{g}$ & 5.5 & 3.6 & 4.7 & $3 \cdot 0,7 \cdot 4$ & $10 \mu \mathrm{g}$ & $7.5 \mu \mathrm{g}$ & $2.5 \mu \mathrm{g}$ & $12 \%$ & $76.1 \%$ & $18 \%$ \\
\hline Vitamin E, mg & $12 \cdot 0$ & $5 \cdot 3$ & $10 \cdot 9$ & $8 \cdot 4,14 \cdot 3$ & $8 \mathrm{mg}$ & $5 \mathrm{mg}$ & $3 \mathrm{mg}$ & $79 \%$ & $2.7 \%$ & $0.5 \%$ \\
\hline Thiamine, mg & 1.5 & 0.6 & 1.4 & $1 \cdot 0,1 \cdot 8$ & $1 \cdot 1 \mathrm{mg}$ & $0.9 \mathrm{mg}$ & $0.5 \mathrm{mg}$ & $71 \%$ & $14.2 \%$ & $0.5 \%$ \\
\hline Riboflavin, mg & 1.9 & $1 \cdot 1$ & 1.7 & $1 \cdot 2,2 \cdot 2$ & $1.3 \mathrm{mg}$ & $1.1 \mathrm{mg}$ & $0.8 \mathrm{mg}$ & $71 \%$ & $18.7 \%$ & $4.3 \%$ \\
\hline Niacin, mg & $23 \cdot 0$ & 13.4 & $20 \cdot 3$ & $14 \cdot 9,27 \cdot 0$ & $15 \mathrm{mg}$ & $12 \mathrm{mg}$ & $9 \mathrm{mg}$ & $75 \%$ & $11.9 \%$ & $2.7 \%$ \\
\hline Vitamin $\mathrm{B}_{6}, \mathrm{mg}$ & $2 \cdot 0$ & 0.8 & 1.8 & $1 \cdot 4,2 \cdot 3$ & $1.2 \mathrm{mg}$ & $1.0 \mathrm{mg}$ & $0.8 \mathrm{mg}$ & $86 \%$ & $6.8 \%$ & $1.8 \%$ \\
\hline Folate, $\mu \mathrm{g}$ & 281 & 121 & 257 & 186,360 & $400 \mu \mathrm{g}$ & $200 \mu \mathrm{g}$ & $100 \mu \mathrm{g}$ & $15 \%$ & $30.6 \%$ & $1.4 \%$ \\
\hline Vitamin $B_{12}, \mu \mathrm{g}$ & $6 \cdot 2$ & 3.5 & 5.5 & $3.7,8.0$ & $2 \mu \mathrm{g}$ & $1.4 \mu \mathrm{g}$ & $1.0 \mu \mathrm{g}$ & $94 \%$ & $2.0 \%$ & $1.6 \%$ \\
\hline Vitamin C, mg & 106.0 & 68.6 & 87.9 & $58.9,132.0$ & $75 \mathrm{mg}$ & $50 \mathrm{mg}$ & $10 \mathrm{mg}$ & $60 \%$ & $16.9 \%$ & 0 \\
\hline Calcium, mg & 802 & 404 & 720 & 532,991 & $800 \mathrm{mg}$ & $500 \mathrm{mg}$ & $400 \mathrm{mg}$ & $40 \%$ & $21.4 \%$ & $11.9 \%$ \\
\hline Phosphorus, mg & 1654 & 638 & 1558 & 1182,1969 & $600 \mathrm{mg}$ & $450 \mathrm{mg}$ & $300 \mathrm{mg}$ & $100 \%$ & 0 & 0 \\
\hline Magnesium, mg & 354 & 134 & 335 & 256,423 & $280 \mathrm{mg}$ & - & - & $68 \%$ & - & - \\
\hline Potassium, g & 3.6 & $1 \cdot 3$ & 3.5 & $2 \cdot 7,4 \cdot 4$ & $3.1 \mathrm{~g}$ & - & $1.6 \mathrm{~g}$ & 0 & - & - \\
\hline Sodium, g & 3.5 & 1.6 & $3 \cdot 2$ & $2 \cdot 3,4 \cdot 2$ & $2.4 \mathrm{~g}$ & - & - & 0 & - & - \\
\hline Iron, mg & 10.5 & 4.0 & $10 \cdot 2$ & $7 \cdot 5,12 \cdot 7$ & $15 \mathrm{mg}$ & $10 \mathrm{mg}$ & $10 \mathrm{mg}^{\mathrm{a}}$ & $12 \%$ & $46.6 \%$ & $46.6 \%$ \\
\hline Zinc, mg & $11 \cdot 1$ & $4 \cdot 1$ & $10 \cdot 6$ & $8 \cdot 2,13.4$ & $7 \mathrm{mg}$ & $5 \mathrm{mg}$ & $4 \mathrm{mg}$ & $86 \%$ & $2.9 \%$ & $0.9 \%$ \\
\hline Copper, mg & 1.2 & 0.5 & 1.2 & $0.8,1.5$ & $0.9 \mathrm{mg}$ & $0.7 \mathrm{mg}$ & $0.4 \mathrm{mg}$ & $97.5 \%$ & $12 \cdot 2 \%$ & $2.5 \%$ \\
\hline Selenium, $\mu \mathrm{g}$ & $47 \cdot 0$ & $22 \cdot 8$ & $43 \cdot 3$ & $30 \cdot 2,60 \cdot 3$ & $50 \mu \mathrm{g}$ & $30 \mu \mathrm{g}$ & $20 \mu \mathrm{g}$ & $38 \%$ & $24.1 \%$ & $8.3 \%$ \\
\hline lodine, $\mu \mathrm{g}$ & 134 & 83 & 114 & 73,165 & $150 \mu \mathrm{g}$ & $100 \mu \mathrm{g}$ & $70 \mu \mathrm{g}$ & $32 \%$ & $42.3 \%$ & $21.6 \%$ \\
\hline
\end{tabular}

The StudentKost study.

NNR: Nordic Nutrition Recommendations; RI: recommended intake; AR: average requirement; LI: lower intake level.

${ }^{a}$ Out of total sample.

${ }^{\mathrm{b}}$ Age group 18-30 was used as the choice of reference intake.

Men had a significantly higher intake of vitamin $\mathrm{B}_{6}$, vitamin $\mathrm{B}_{12}$ and vitamin $\mathrm{E}$, iodine and calcium than women. Women, on the other hand, had a significantly higher intake of vitamin $\mathrm{C}$ than men. Importantly, the listed intakes do not include the use of supplements. Participants' use of supplements is described below.

In this study, $88 \%$ of women and $85 \%$ of men did not reach the RI of vitamin D. The RI of vitamin A was not

Table 5. Micronutrient intake per day compared to NNR and related to recommendations in percentage

Men

\begin{tabular}{|c|c|c|c|c|c|c|c|c|c|c|}
\hline \multirow[b]{2}{*}{ Micronutrients } & \multirow[b]{2}{*}{ Mean } & \multirow[b]{2}{*}{ SD } & \multirow[b]{2}{*}{ Median } & \multirow[b]{2}{*}{$25^{\text {th }}-75^{\text {th }}$ percentile } & \multicolumn{3}{|c|}{ NNR2012 recommendation } & \multicolumn{3}{|c|}{ Percentage of participants ${ }^{a}$} \\
\hline & & & & & $R \mathrm{I}^{\mathrm{b}}$ & $\mathrm{AR}^{\mathrm{b}}$ & $\mathrm{LI}^{\mathrm{b}}$ & $\geq \mathrm{RI}$ & $<\mathrm{AR}$ & $<\mathrm{LI}$ \\
\hline Vitamin A, $\mu \mathrm{g}$ & 963 & 779 & 762 & 463,1137 & $900 \mu \mathrm{g}$ & $600 \mu \mathrm{g}$ & $500 \mu \mathrm{g}$ & $39 \%$ & $36.5 \%$ & $28.1 \%$ \\
\hline Vitamin D, $\mu \mathrm{g}$ & $6 \cdot 0$ & 3.7 & 5.5 & $3 \cdot 3,8 \cdot 0$ & $10 \mu \mathrm{g}$ & $7.5 \mu \mathrm{g}$ & $2.5 \mu \mathrm{g}$ & $15 \%$ & $68.5 \%$ & $14 \%$ \\
\hline Vitamin E, mg & $13 \cdot 3$ & 6.0 & $12 \cdot 3$ & $9 \cdot 0,16 \cdot 8$ & $10 \mathrm{mg}$ & $6 \mathrm{mg}$ & $4 \mathrm{mg}$ & $70 \%$ & $7.9 \%$ & $1.7 \%$ \\
\hline Thiamine, mg & 1.6 & 0.6 & 1.6 & $1 \cdot 1,2 \cdot 0$ & $1.4 \mathrm{mg}$ & $1.2 \mathrm{mg}$ & $0.6 \mathrm{mg}$ & $58 \%$ & $31.5 \%$ & $1.7 \%$ \\
\hline Riboflavin, mg & $2 \cdot 2$ & $1 \cdot 1$ & $2 \cdot 0$ & $1 \cdot 5,2 \cdot 6$ & $1.6 \mathrm{mg}$ & $1.4 \mathrm{mg}$ & $0.8 \mathrm{mg}$ & $73 \%$ & $19.1 \%$ & $4.5 \%$ \\
\hline Niacin, mg & $26 \cdot 3$ & $13 \cdot 1$ & $24 \cdot 6$ & $17 \cdot 8,31 \cdot 4$ & $19 \mathrm{mg}$ & $15 \mathrm{mg}$ & $12 \mathrm{mg}$ & $73 \%$ & $14.0 \%$ & $6.7 \%$ \\
\hline Vitamin $\mathrm{B}_{6}, \mathrm{mg}$ & $2 \cdot 2$ & 0.8 & $2 \cdot 0$ & $1 \cdot 6,2 \cdot 6$ & $1.5 \mathrm{mg}$ & $1.3 \mathrm{mg}$ & $1.0 \mathrm{mg}$ & $80 \%$ & $13.5 \%$ & $5.1 \%$ \\
\hline Folate, $\mu \mathrm{g}$ & 275 & 119 & 265 & 188,346 & $300 \mu \mathrm{g}$ & $200 \mu \mathrm{g}$ & $100 \mu \mathrm{g}$ & $37 \%$ & $28.7 \%$ & $2.8 \%$ \\
\hline Vitamin $\mathrm{B}_{12}, \mu \mathrm{g}$ & $7 \cdot 2$ & 3.7 & 6.5 & $4 \cdot 5,9 \cdot 1$ & $2 \mu \mathrm{g}$ & $1.4 \mu \mathrm{g}$ & $1.0 \mu \mathrm{g}$ & $96 \%$ & $1.7 \%$ & $1.1 \%$ \\
\hline Vitamin C, mg & 88.5 & 57.5 & 73.4 & $48.6,113.5$ & $75 \mathrm{mg}$ & $60 \mathrm{mg}$ & $10 \mathrm{mg}$ & $48 \%$ & $37.6 \%$ & $0.6 \%$ \\
\hline Calcium, mg & 887 & 443 & 790 & 568,1088 & $800 \mathrm{mg}$ & $500 \mathrm{mg}$ & $400 \mathrm{mg}$ & $49 \%$ & $20.2 \%$ & $11.2 \%$ \\
\hline Phosphorus, mg & 1805 & 665 & 1779 & 1265,2245 & $600 \mathrm{mg}$ & $450 \mathrm{mg}$ & $300 \mathrm{mg}$ & $99 \%$ & 0 & 0 \\
\hline Magnesium, mg & 362 & 140 & 344 & 255,438 & $350 \mathrm{mg}$ & - & - & $49 \%$ & - & - \\
\hline Potassium, g & 3.7 & 1.4 & 3.6 & $2 \cdot 6,4 \cdot 3$ & $3.5 \mathrm{~g}$ & - & $1.6 \mathrm{~g}$ & 0 & - & - \\
\hline Sodium, g & $4 \cdot 1$ & 1.8 & 4.0 & $2 \cdot 8,5 \cdot 0$ & $2.4 \mathrm{~g}$ & - & - & 0 & - & - \\
\hline Iron, mg & $11 \cdot 0$ & 4.3 & $10 \cdot 9$ & $8.0,14.0$ & $9 \mathrm{mg}$ & $7 \mathrm{mg}$ & $7 \mathrm{mg}$ & $62 \%$ & $18.5 \%$ & $18.5 \%$ \\
\hline Zinc, mg & $12 \cdot 7$ & 4.5 & $12 \cdot 8$ & $9 \cdot 2,15 \cdot 6$ & $9 \mathrm{mg}$ & $6 \mathrm{mg}$ & $5 \mathrm{mg}$ & $77 \%$ & $5.1 \%$ & $1.7 \%$ \\
\hline Copper, mg & $1 \cdot 2$ & 0.5 & $1 \cdot 1$ & $0.8,1.5$ & $0.9 \mathrm{mg}$ & $0.7 \mathrm{mg}$ & $0.4 \mathrm{mg}$ & $70 \%$ & $13.5 \%$ & $2.3 \%$ \\
\hline Selenium, $\mu \mathrm{g}$ & 54.6 & $24 \cdot 2$ & 52.7 & $38 \cdot 6,67 \cdot 7$ & $60 \mu \mathrm{g}$ & $35 \mu \mathrm{g}$ & $20 \mu \mathrm{g}$ & $37 \%$ & $18.0 \%$ & $6.2 \%$ \\
\hline lodine, $\mu \mathrm{g}$ & 143 & 78 & 133 & 91,184 & $150 \mu \mathrm{g}$ & $100 \mu \mathrm{g}$ & $70 \mu \mathrm{g}$ & $41 \%$ & $33.1 \%$ & $16.9 \%$ \\
\hline
\end{tabular}

The StudentKost study.

NNR: Nordic Nutrition Recommendations; RI: recommended intake; AR: average requirement; LI: lower intake level.

${ }^{\text {a }}$ Out of total sample.

b Age group of 18-30 years was used as the choice of reference intake. 
reached by $48 \%$ women and $61 \%$ of men. Approximately 85 $\%$ of female students did not reach the RI of folate, $88 \%$ for iron and $68 \%$ for iodine. Regarding male students, a total of $63 \%$ did not reach the RI of folate, $38 \%$ for iron and $59 \%$ for iodine. The Norwegian Directorate of Health recommends reducing the salt intake to a maximum of $5 \mathrm{~g} / \mathrm{d}^{(1)}$, while the NNR recommends a maximum of $6 \mathrm{~g} / \mathrm{d}^{(11)}$. In this sample, both genders consumed more salt than recommended, with a mean intake of $8.7 \mathrm{~g} / \mathrm{d}$ among women and $10.2 \mathrm{~g} / \mathrm{d}$ among men.

Probability of inadequate micronutrient intake. We also assessed the proportions of participants with minimal probability of inadequate intake (total intake of the respective micronutrient at or above the recommended intake), relatively bigh probability of inadequate intake (the total intake of the respective micronutrient lower than the average intake) and very high probability of inadequate intake (the total intake of the respective nutrient below the lower intake), respectively (Tables 4 and 5). The probability of inadequate intake was low for vitamin E, B-vitamins other than folate, phosphorus, zinc, copper, riboflavin, and niacin (proportion of students with intake at or above RI $\geq 70 \%$ ) (Tables 4 and 5). The proportion of female participants with intake less than the average requirement, and thus, relatively high probability of inadequate micronutrient intakes was substantial for vitamin A $(30 \%)$, vitamin D $(76 \%)$, folate (31\%), calcium (21\%), iron (47\%), selenium (24\%) and iodine $(42 \%)$ (Table 4). A smaller, but still substantial, proportion of female students had a very high probability of inadequate intake of vitamin A $(16 \%)$, vitamin D $(18 \%)$, iron $(47 \%)$ and iodine $(22 \%)$. The proportion of male participants with a relatively high probability of inadequate micronutrient intakes was substantial for vitamin A $(37 \%)$, vitamin C (38\%), vitamin D (69\%), folate (29\%), calcium $(20 \%)$ and iodine $(33 \%)$ (Table 5). A smaller but still substantial proportion of male students had a very high probability of inadequate intake of vitamin A (28\%), vitamin D $(14 \%)$, iron $(19 \%)$ and iodine $(17 \%)$.

\section{Supplements}

A total of $17 \cdot 6 \%$ female and $16.3 \%$ male students reported taking omega- 3 or cod-liver oil capsules every day. In this sample, $16.9 \%$ of women and $12.4 \%$ of men reported taking multivitamins, or multivitamins with minerals, every day. Although folate intake was lower than recommended for women, only $2.5 \%$ of the sample reported taking folate supplements every day. Furthermore, $95 \%$ of all women reported never taking folate supplements. Men showed similar numbers with $96 \%$ never taking folate supplements, and $2 \%$ taking it every day. Similarly, only $6 \%$ of women took iron supplements every day, while $4 \%$ of men did the same. Among vitamin A, C and D supplements, vitamin D supplements were consumed most frequently with $12 \%$ of both women and men taking it every day.

\section{Discussion}

Our main findings are that college students, both male and female, have a somewhat suboptimal diet compared to Norwegian dietary guidelines. Fruits, vegetables, fish, milk and whole-grain products are all significant contributors to a varied and healthy diet, especially during the preconception period $^{(27)}$. Fruit and vegetable intake was substantially lower than recommended and a lot lower than reported in Norkost $3^{(2)}$. The consumption of both whole grain and fish were low for both genders, with mean intake just above half of the recommended intake of fish. This may partly explain the low vitamin $\mathrm{D}$ and iodine intake.

WHO estimates that approximately 1.7 million or $2.8 \%$ of deaths worldwide are related to low consumption of fruit and vegetables $^{(28)}$, and low fruit consumption was the most significant individual dietary risk factor in $2010^{(29)}$. A high intake of fruit and vegetables has been associated with a reduced risk of Cardiovascular disease (CVD), cancer and other major health problems ${ }^{(30-32)}$. Eating fish 2-4 times a week may reduce the risk of heart conditions such as Coronary heart disease (CHD) and heart failure ${ }^{(33,34)}$. According to the Global Burden of Disease study from 2019, a high intake of sodium, and a low intake of whole grains and fruits were the leading dietary risk factors for death and disability-adjusted life years, of which low intake of whole grain was the leading risk among young adults ${ }^{(35)}$. Dietary fibre and whole-grain products connect to a multitude of health benefits, among them being a lower risk of high blood pressure and a lower concentration of Low-density lipoprotein-cholesterol (LDL), reduced risk of all-cause mortality as well as cardiovascular disease and cancer mortality ${ }^{(36-38)}$, of which high blood pressure before pregnancy is considered a risk factor for pre-eclampsia ${ }^{(39)}$. Milk and dairy products contribute between 60 and $80 \%$ of the iodine intake in the Norwegian diet and are considered its most important source ${ }^{(40)}$.

Only $12 \%$ complied with the recommendation of '5-A-Day', or $500 \mathrm{~g} / \mathrm{d}$, even when juice was included. In Norkost 3, from the entire sample, a total of $25 \%$ of women and $22 \%$ of men complied with the recommendation on fruit and vegetables ${ }^{(2)}$, while results from the British National Diet and Nutrition Survey (NDNS) showed that $32 \%$ women and $29 \%$ men aged 19-64 satisfied the '5-A-Day' recommendation ${ }^{(41)}$. Results from the Dutch National Food Consumption Survey (DNFCS), however, showed that among 703 participants aged 19-30 years, less than $5 \%$ satisfied the recommended $200 \mathrm{~g}$ vegetables per day $^{(42)}$. Consuming $500 \mathrm{~g}$ or more fruit and vegetables per day has been associated with reduced risk of cardiovascular diseases $^{(43)}$, such as coronary heart disease ${ }^{(44)}$, and Jian et al. found the lowest risk for cardiovascular disease among those consuming $800 \mathrm{~g}$ or more fruit and vegetables per day ${ }^{(43)}$.

Macronutrient contribution to energy intake seems to be in line with the recommendations from the NNR within a few percentages $^{(11)}$. While sugar intake was well within recommended limits for most participants, one unfavourable finding was the low intake of dietary fibre for both genders. Countless studies have investigated the importance of dietary fibre, of 
which many have found inverse associations with risk of CVD and all cancers ${ }^{(45-47)}$. Dietary fibre also promotes a healthy microbiota ${ }^{(48,49)}$. These findings encourage further investigation into young adults' diet as an important determinant for long-term health.

In this limited sample of college students, we found high proportions of participants with a relatively high probability of inadequate intake of vitamin A, vitamin D, vitamin C (male), folate, calcium, iron, selenium and iodine. The suboptimal intake of these nutrients is a direct consequence of the low intake of core foods such as fruits, vegetables, whole grain, fish and dairy products in the present study. However, a low intake does not translate directly to compromised nutritional status of the particular micronutrients, this being especially relevant to vitamin $\mathrm{D}$ status since vitamin $\mathrm{D}$ can be synthesized in the skin by the ultraviolet radiation of its precursor 7-dehydrocholesterol ${ }^{(50,51)}$. Intake of folate, iron and iodine was suboptimal among female students. Folate (vitamin $\mathrm{B}_{9}$ ) is considered a critical nutrient during the periconception per$\operatorname{iod}^{(52,53)}$, as a low intake has been associated with increased risk of Spina Bifida and other neural tube defects for the foetus ${ }^{(54)}$. Sufficient iron intake during preconception and reproductive years are vital in building capacity for the large requirements during pregnancy and the inherent risk of iron deficiency and anaemia ${ }^{(55)}$. Lastly, a low intake of iodine increases the risk of goitre, and a sufficient intake is necessary to ensure proper foetal development from early pregnancy onwards ${ }^{(56)}$. Iodine also has essential roles in several bodily regulatory functions, like metabolic rates, the immune system and the central nervous system ${ }^{(57)}$.

We found gender differences in diet among students, with female students in general eating healthier than male students. This raises concern about male awareness of the importance of diet, as new research also highlights the importance of a healthy paternal diet during the preconception period ${ }^{(4-6)}$. We expected some gender differences due to differences in energy intake, but the higher absolute intake of fruit and vegetables, whole grain, dietary fibre and proportion of energy from carbohydrates among women were surprising. Results from Norkost 3 and the DNFCS showed no significant difference in the intake of vegetables between genders, while this study showed a difference in favour of women ${ }^{(2)}$.

\section{Study strengths and limitations}

This study aimed to assess the dietary intake and nutritional adequacy of young adults through a dietary questionnaire, here represented by students in their preconception years. The questionnaire was designed so that participants had to answer all questions, except height, weight and their email address. The advantage of using this method was that we got very few missing values in our data. On the other hand, we do not know what questions caused the remaining 121 participants not to complete the survey. Using an FFQ has its pros and cons. The FFQ was very comprehensive, and we were able to collect a large amount of information in a short period, but as the food items are set beforehand, we missed some diet variables which could have had an impact on our results. Most noticeably, specific vegetables and berries, which were mentioned by a good number of participants in the comment section on the end of each part of the questionnaire. Misreporting in dietary surveys is common ${ }^{(58)}$. If overreporting of healthy foods or underreporting of unhealthy foods are prevalent in the present study, challenges related to diet may be even more substantial than our findings suggest, and public health initiatives aimed at diets in this population of young adults would be even more relevant. Red meat intake may be underestimated because we did not include taco in the calculation, which is a popular dish that is eaten by $13 \%$ of the population at least once a week ${ }^{(59)}$. As a consequence, we cannot make definitive conclusions on meat intake, which might have an impact on iron intake.

There might also be some limitations in the software that was used. It is difficult to convert the number of portions to a total of grams eaten, as the calculations are based on a report on standard portion sizes from the Norwegian Food Safety Authority, the University of Oslo, and the Norwegian Directorate of Health ${ }^{(60)}$. How you define one portion is based on your personal preference, so this leaves room for error. The portions used are the average portions sized for adults in Norway ${ }^{(60)}$.

We contacted all enrolled university students by way of their institutional email in the recruitment to this study; hence, the low response rate compromises the generalisability of our findings. Although all students received an email, we do not know if they check their institutional email consistently. We do not know to which degree the diet of our study population differs from the rest of the accessible population at UiA or students at other universities in Norway. Moreover, the sample might not be an accurate representation of the entire young adult population which must be considered when interpreting this data. Still, the best data for this age group in Norway are currently from the national dietary surveys, including 18-70 years, with only 281 participants being $18-29$ years old, as well as being from $2011^{(2)}$.

Since we targeted students, educational attainment in this sample is at a higher percentage than the average of the Norwegian population, where $33.4 \%$ aged 16 years or older have attained higher education ${ }^{(61)}$. However, it is similar to the education level of the national study Norkost $3^{(2)}$. As there are a lot more women than men in the sample, the data on women may be more representative than the data on men. If anything, due to education attainment and selfselection for the study, our sample is likely to be more healthconscious than the broader population, making our findings regarding dietary weaknesses of concern in a public health perspective.

\section{Conclusion}

This study indicates dietary weaknesses among university students and potential inadequacy for several micronutrients. Gender differences indicated a healthier diet in favour of female students, including higher consumption of fruits, vegetables, and fibre, and lower red meat and high energy-dense dairy products consumption. A substantial proportion of 
both male and female students had a relatively high probability of inadequate intake of several micronutrients, including vita$\min \mathrm{A}$, vitamin $\mathrm{D}$, folate, iron and iodine.

Our findings have limitations and should be interpreted with caution. However, the fact that our sample might be a selected group with an above-average interest in diet and nutrition highlights the need for attention and public health nutrition actions towards this group. Our results call for more representative dietary assessment studies to be performed among young adults to inform further action towards a healthier diet during the preconception period.

\section{Acknowledgements}

We would like to thank Lorentz Salvesen and Ida U. Valand for their contribution to this project. Lorentz conducted the nutrition calculation, and Ida was involved in the recruitment process.

This work was supported by the University of Agder Priority Research Center on Lifecourse Nutrition.

There are no conflicts of interest.

The authors assert that all procedures contributing to this work comply with the ethical standards of the Helsinki Declaration of 1975, as revised in 2008, and have been approved by the Institutional Ethics Committee and the Norwegian Centre for Research Data.

E.L.V., N.C.Ø., D.E. and E.R.H. invented and designed the study. E.L.V. carried out the study, analysed data and drafted the paper. N.C.Ø., D.E. and E.R.H. contributed to the interpretation of the findings. All authors critically read and approved the final version.

\section{References}

1. Norwegian Nutrition Council (2011) Kostråd for å fremme folkehelsen og forebygge kroniske sykdommer - Metodologi og vitenskapelig kunnskapsgrunnlag. Oslo: Norwegian Directorate of Health.

2. Totland TH, Melnæs BK, Lundberg-Hallén N, et al. (2012) Norkost 3 En landsomfattende kostholdsundersokelse blant menn og kvinner $i$ Norge $i$ alderen 18-70 arr, 2010-11. Oslo: Norwegian Directorate of Health.

3. Johansson L \& Solvoll K (1999) NORKOST 1997 Landsomfattende kostboldsundersokelse blant menn og kvinner $i$ alderen 16-79 år. Oslo: Statens råd for ernæring og fysisk aktivitet.

4. Stephenson J, Heslehurst N, Hall J, et al. (2018) Before the beginning: nutrition and lifestyle in the preconception period and its importance for future health. Lancet 391, 1830-1841.

5. Fleming TP, Watkins AJ, Velazquez MA, et al. (2018) Origins of lifetime health around the time of conception: causes and consequences. Lancet 391, 1842-1852.

6. Barker M, Dombrowski SU, Colbourn T, et al. (2018) Intervention strategies to improve nutrition and health behaviours before conception. Lancet 391, 1853-1864.

7. Nätt D, Kugelberg U, Casas E, et al. (2019) Human sperm displays rapid responses to diet. PLoS Biol 17, e3000559.

8. Frey KA, Navarro SM, Kotelchuck M, et al. (2008) The clinical content of preconception care: preconception care for men. Am J Obstet Gynecol 199, 6 Suppl. 2, S389-S395.

9. Norwegian Directorate of Health (2019) Kosthold for gravide. https:// helsenorge.no/gravid/mat-og-drikke-nar-du-er-gravid\#Viktigevitaminer-og-mineraler-for-gravide-

10. Statistics Norway (2019) Students in higher education. https://www. ssb.no/en/utdanning/statistikker/utuvh (updated 28 March 2019).
11. Nordic Council of Ministers (2014) Nordic Nutrition Recommendations 2012, 5th ed. Copenhagen: Nordic Council of Ministers.

12. Statistics Norway (2020) Births Statistics Norway. https://www.ssb. no/en/befolkning/statistikker/fodte/aar

13. Salvesen L, Hillesund ER, Vik FN, et al. (2019) Reproducibility and relative validity of a newly developed web-based food-frequency questionnaire for assessment of preconception diet. BMC Nutr $5,47$.

14. Ramboll (2019). SurveyXact. https://www.surveyxact.com/

15. Python (2019) Python. https://www.python.org/

16. Lauritsen J (2019) FoodCalc. http://www.ibt.ku.dk/jesper/foodcalc/

17. Matportalen (2018) The Norwegian Food Composition Table. http://www.matportalen.no/verktoy/

the_norwegian_food_composition_table/

18. Norwegian Directorate of Health (2014) Anbefalinger om kosthold, ernaring og fysisk aktivitet. 04.02.2019. Report No.: IS-2170.

19. IBM (2019) IBM SPSS software. https://www.ibm.com/analytics/ spss-statistics-software

20. Axa (2018) Gold Energi Müsli. https://www.axa.no/produkter/ musli/gold-musli-energi/

21. Matportalen (2018) Matvaretabellen. http://www.matvaretabellen. no/?language $=$ en

22. Orkla Foods Norge (2019) Hamburger med brød 4 stk. http:// produktkatalog.orklafoods.no/dagligvare/produktkatalog/produkter/ Middag/Hamburger/Basic/1756/

23. Orkla Foods Norge (2019) Stor pai med ost \& skinke. https://produktkatalog.orklafoods.no/dagligvare/produktkatalog/produkter/ Middag/Ferdigretter/Paier/5896_5898/

24. Meny (2019) Lapskaus lys. https://meny.no/varer/middag/ ferdigmaltider/gryterett-ferdig/lapskaus-lys-7035620000089

25. Meny (2019) Lasagne. https://meny.no/varer/middag/ferdigmaltider/ lasagne-ferdig/lasagne-7035620019661

26. Kolonial.no (2019) Lapskaus 500 g. https://www.grandiosa.no/ products/grandiosa-original/

27. Tommy's (2018) Tips for pre-pregnancy diet Tommy's. https:// www.tommys.org/pregnancy-information/planning-pregnancy/ are-you-ready-conceive/tips-healthy-pre-pregnancy-diet (updated 05 June 2018; cited 25 June 2020).

28. World Health Organization (2019) Promoting fruit and vegetable consumption around the world. https://www.who.int/dietphysicalactivity/ fruit/en/index $2 . h t m l$

29. Lim SS, Vos T, Flaxman AD, et al. (2012) A comparative risk assessment of burden of disease and injury attributable to 67 risk factors and risk factor clusters in 21 regions, 1990-2010: a systematic analysis for the Global Burden of Disease Study 2010. Lancet 380, 2224-2260.

30. Slavin JL \& Lloyd B (2012) Health benefits of fruits and vegetables. Adv Nutr 3, 506-516.

31. Pem D \& Jeewon R (2015) Fruit and vegetable intake: benefits and progress of nutrition education interventions-narrative review article. Iran J Public Health 44, 1309-1321.

32. Yip CSC, Chan W \& Fielding R (2019) The associations of fruit and vegetable intakes with burden of diseases: a systematic review of meta-analyses. J Acad Nutr Diet 119, 464-481.

33. Zheng J, Huang T, Yu Y, et al. (2012) Fish consumption and CHD mortality: an updated meta-analysis of seventeen cohort studies. Public Health Nutr 15, 725-737.

34. Djousse L, Akinkuolie AO, Wu JH, et al. (2012) Fish consumption, omega- 3 fatty acids and risk of heart failure: a meta-analysis. Clin Nutr 31, 846-853.

35. GBD 2017 Diet Collaborators (2019) Health effects of dietary risks in 195 countries, 1990-2017: a systematic analysis for the Global Burden of Disease Study 2017. Lancet 393, 1958-1972.

36. Aune D, Keum N, Giovannucci E, et al. (2016) Whole grain consumption and risk of cardiovascular disease, cancer, and all cause and cause specific mortality: systematic review and dose-response meta-analysis of prospective studies. BMJ 353, 1-14.

37. Zhang B, Zhao Q, Guo W, et al. (2018) Association of whole grain intake with all-cause, cardiovascular, and cancer mortality: a 
systematic review and dose-response meta-analysis from prospective cohort studies. Eur J Clin Nutr 72, 57-65.

38. Chen GC, Tong X, Xu JY, et al. (2016) Whole-grain intake and total, cardiovascular, and cancer mortality: a systematic review and meta-analysis of prospective studies. Am J Clin Nutr 104, $164-172$.

39. NHS (2018) Pre-eclampsia NHS: NHS. https://www.nhs.uk/ conditions/pre-eclampsia/

40. Norwegian Nutrition Council (2016) Risiko for jodmangel $i$ Norge. Oslo: Norwegian Directorate of Health. Report No.: IS-0591.

41. Public Health England (2018) National Diet and Nutrition Survey. London, UK. https://www.gov.uk/government/statistics/ndnsresults-from-years-7-and-8-combined

42. van Rossum CTM, Franse HP, Verkaik-Kloosterman J, et al. (2011) Dutch National Food Consumption Survey 2007-2010. Haag: Ministry of Health, Welfare and Sports. Report No.: 350050006/2011.

43. Zhan J, Liu YJ, Cai LB, et al. (2017) Fruit and vegetable consumption and risk of cardiovascular disease: A meta-analysis of prospective cohort studies. Crit Rev Food Sci Nutr 57, 1650-1663.

44. Gan Y, Tong X, Li L, et al. (2015) Consumption of fruit and vegetable and risk of coronary heart disease: a meta-analysis of prospective cohort studies. Int J Cardiol 183, 129-137.

45. Stephen AM, Champ MM, Cloran SJ, et al. (2017) Dietary fibre in Europe: current state of knowledge on definitions, sources, recommendations, intakes and relationships to health. Nutr Res Rev 30, 149-190.

46. Chan CW \& Lee PH (2016) Association between dietary fibre intake with cancer and all-cause mortality among 15740 adults: the National Health and Nutrition Examination Survey III. J Hum Nutr Diet 29, 633-642.

47. Kim Y \& Je Y (2016) Dietary fibre intake and mortality from cardiovascular disease and all cancers: a meta-analysis of prospective cohort studies. Arch Cardiovasc Dis 109, 39-54.

48. Tap J, Furet JP, Bensaada M, et al. (2015) Gut microbiota richness promotes its stability upon increased dietary fibre intake in healthy adults. Environ Microbiol 17, 4954-4964.
49. Makki K, Deehan EC, Walter J, et al. (2018) The impact of dietary fiber on gut microbiota in host health and disease. Cell Host Microbe 23, 705-715.

50. Dahl SR \& Thorsby PM (2014) [How to measure vitamin D status?]. Tidssker Nor Laegeforen 134, 729-731.

51. Nair R \& Maseeh A (2012) Vitamin D: The "sunshine" vitamin. J Pharmacol Pharmather 3, 9.

52. Gomes S, Lopes C \& Pinto E (2016) Folate and folic acid in the periconceptional period: recommendations from official health organizations in thirty-six countries worldwide and WHO. Public Health Nutr 19, 176-189.

53. World Health Organization (2019) Periconceptional folic acid supplementation to prevent neural tube defects. https://www.who.int/elena/titles/ folate_periconceptional/en/

54. Bailey LB, Stover PJ, McNulty H, et al. (2015) Biomarkers of nutrition for development-folate review. J Nutr 145, 1636s-1680s.

55. InformedHealth.org [Internet] (2009) Pregnancy and Birth: Do All Pregnant Women Need To Take Iron Supplements?. Cologne, Germany: Institute for Quality and Efficiency in Health Care. https://www.ncbi.nlm.nih.gov/books/NBK279574/ (updated 22.03.2018).

56. Niwattisaiwong S, Burman KD \& Li-Ng M (2017) Iodine deficiency: clinical implications. Cleve Clin J Med 84, 236-44.

57. National Institutes of Health (2019) Iodine: fact sheet for health professionals. https://ods.od.nih.gov/factsheets/Iodine-HealthProfessional/

58. Willett W (2013) Nutritional Epidemiology, 3rd ed., Oxford: Oxford University Press.

59. Ipsos (2018) Norske Spisefakta 2018: Frokost hver dag - taco hver fredag. https://www.ipsos.com/nb-no/norske-spisefakta2018-frokost-hver-dag-taco-hver-fredag

60. Dalane JØ, Bergvatn TM, Kielland E, et al. (2015) Mål, vekt og porsjonsstorrelser for matvarer. Oslo: Norwegian Food Safety Authority, University of Oslo, Norwegian Directorate of Health.

61. Statistics Norway (2018) Educational Attainment of the Population. https://www.ssb.no/en/utdanning/statistikker/utniv 